EPJ Web of Conferences 47, 17001 (2013)

DOI: $10.1051 /$ epjconf/20134717001

(C) Owned by the authors, published by EDP Sciences, 2013

\title{
The APACHE survey hardware and software design: Tools for an automatic search of small-size transiting exoplanets
}

\author{
Jean-Marc Christille ${ }^{1,5, a}$, A. Bernagozzi ${ }^{1}$, E. Bertolini ${ }^{1}$, P. Calcidese ${ }^{1}$, \\ A. Carbognani ${ }^{1}$, D. Cenadelli ${ }^{1}$, M. Damasso ${ }^{1,2,4}$, P. Giacobbe ${ }^{1,3,4}$, L. Lanteri ${ }^{4}$, \\ M.G. Lattanzi ${ }^{4}$, A. Sozzetti ${ }^{4}$ and R. Smart ${ }^{4}$ \\ ${ }^{1}$ Astronomical Observatory of the Autonomous Region of the Aosta Valley, Loc. Lignan 39, \\ 11020 Nus (Aosta), Italy \\ ${ }^{2}$ Dept. of Physics and Astronomy, University of Padova, Vicolo dell'Osservatorio 5, \\ 35122 Padova, Italy \\ ${ }^{3}$ Dept. of Physics, University of Trieste, via Tiepolo 11, 34143 Trieste, Italy \\ ${ }^{4}$ INAF - Astrophysical Observatory of Torino, via Osservatorio 20, 10025 Pino Torinese, Italy \\ ${ }^{5}$ Dept. of Physics, University of Perugia, via A. Pascoli, 06123 Perugia, Italy
}

\begin{abstract}
Small-size ground-based telescopes can effectively be used to look for transiting rocky planets around nearby low-mass $\mathrm{M}$ stars using the photometric transit method, as recently demonstrated for example by the MEarth project. Since 2008 at the Astronomical Observatory of the Autonomous Region of Aosta Valley (OAVdA), we have been preparing for the long-term photometric survey APACHE, aimed at finding transiting small-size planets around thousands of nearby early and mid-M dwarfs. APACHE (A PAthway toward the Characterization of Habitable Earths) is designed to use an array of five dedicated and identical 40-cm Ritchey-Chretien telescopes and its observations started at the beginning of summer 2012. The main characteristics of the survey final set up and the preliminary results from the first weeks of observations will be discussed.
\end{abstract}

\section{THE PRESENT STATUS OF THE APACHE SURVEY, A COLLABORATION BETWEEN INAF-OSSERVATORIO ASTROFISICO DI TORINO AND THE ASTRONOMICAL OBSERVATORY OF THE AUTONOMOUS REGION OF THE AOSTA VALLEY (OAVDA). E. BERTOLINI AND M.G. LATTANZI: PROJECT MANAGERS, A. SOZZETTI: PROJECT SCIENTIST}

The APACHE observations started in June 2012 using four identical 40-cm RC telescopes located on the renewed scientific platform of OAVdA.The movements of the telescopes and the data acquisition operations are fully autonomous and controlled by the open source software RTS2 (http://rts2 . org), which we have adapted to the needs of the APACHE survey: the system, accessing to a customized database, takes images, ensures a good pointing (i.e. the target star is always placed at the centre of the CCD sensor),keeps track of what was done. Each telescope observes stars selected from one of the four sub-performed by the upgraded pipeline TEEPEE developed by the authors and described in Damasso et al. [1]. TEEPEE performs differential aperture photometry testing several apertures and choosing the best one together with the best set of comparison stars. The last TEEPEE release has implemented new features in order to manage the whole data reduction according to the big amount of raw data and

${ }^{a}$ e-mail: jeanmarc.christille@gmail.com

This is an Open Access article distributed under the terms of the Creative Commons Attribution License 2.0, which permits unrestricted use, distribution, and reproduction in any medium, provided the original work is properly cited. 

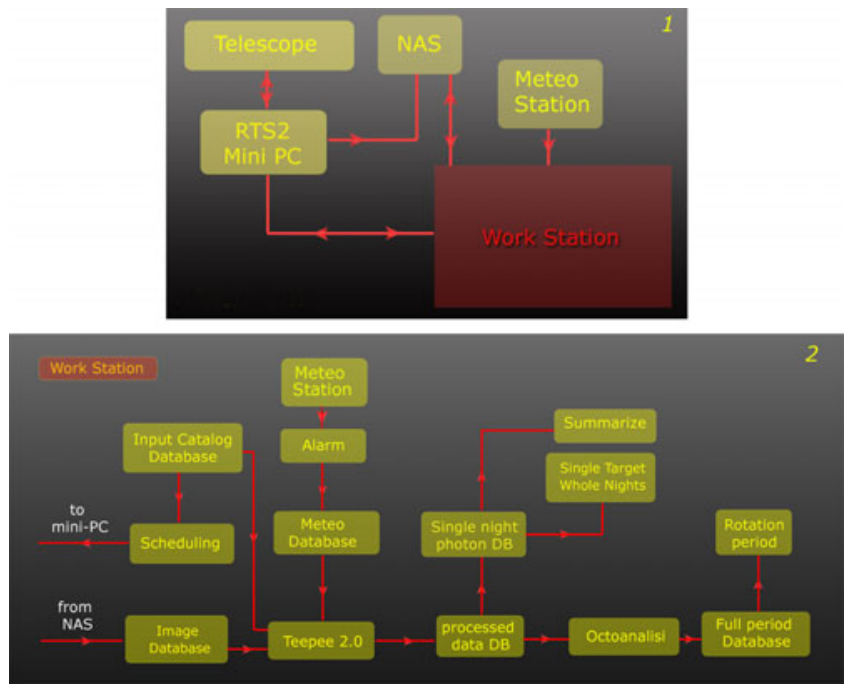

Figure 1. Here we show, in the picture 1 and 2, the block diagram of our data reduction pipeline called TEEPEE2.0 for the photometric study of each target in a single night. In addition, the full period analisys routine is called OCTO. Every result is inserted in our database tables.

the observations strategy. The main feature of this new release is the sistematic organization within a database of the whole results of the pipeline. Each result of every process (from raw to photometrized data) during the data reduction is stored in a customized database. Moreover we are developing analysis pipeline fully automatized based on the DB system in order to automatize even the noise-analysis and the periodic signal search as described in Fig. 1. We are now developing the pipeline TSE (TEEPEE SEntinel) which is designed to perform real-time differential photometry targets during each observing night. The APACHE project has an official web site: http://apacheproject.altervista.org/.

\section{DEFINING THE APACHE OBSERVING STRATEGY AND THE INPUT CATALOGUE OF RED DWARFS}

The results from the pilot study helped us to define a preliminary observing strategy for the targeted survey APACHE. Taking into account the use of synthetic transits, by assuming different numbers of consecutive exposures (from 1 to 4 ) and different temporal samplings (from 20 to 50 minutes), we evaluated the transit detection probabilities for stars with different average phase coverages. The temporal sampling is here defined as the time interval between two consecutive pointings of the telescope to the same target.The results of this analysis are showed in the plot on the left. At the moment, we decided to adopt the strategy of 3 exposures every 20 minutes, which will be eventually revisited after the first season of the survey. We defined the APACHE Input Catalogue (AIC) of red dwarfs starting from the list of 8889 stars in Lepine et Gaidos [2]. The AIC is composed of 3000 targets selected on the base of their visibility from our site and a suitable number of potentially good comparison stars in the f.o.v. Moreover, several catalogues have been cross-checked to get information about the targets, as a precise determination of spectral class, their projected rotational velocity vsini, their level of chromospheric activity, and excluding known spectroscopic binary systems. The number of the Gaia transits scheduled for each target has been also determined, and stars with $>100$ Gaia observations will be prioritized. On the base of all the information collected, a final ranking has been determined to define the priority for observations. 

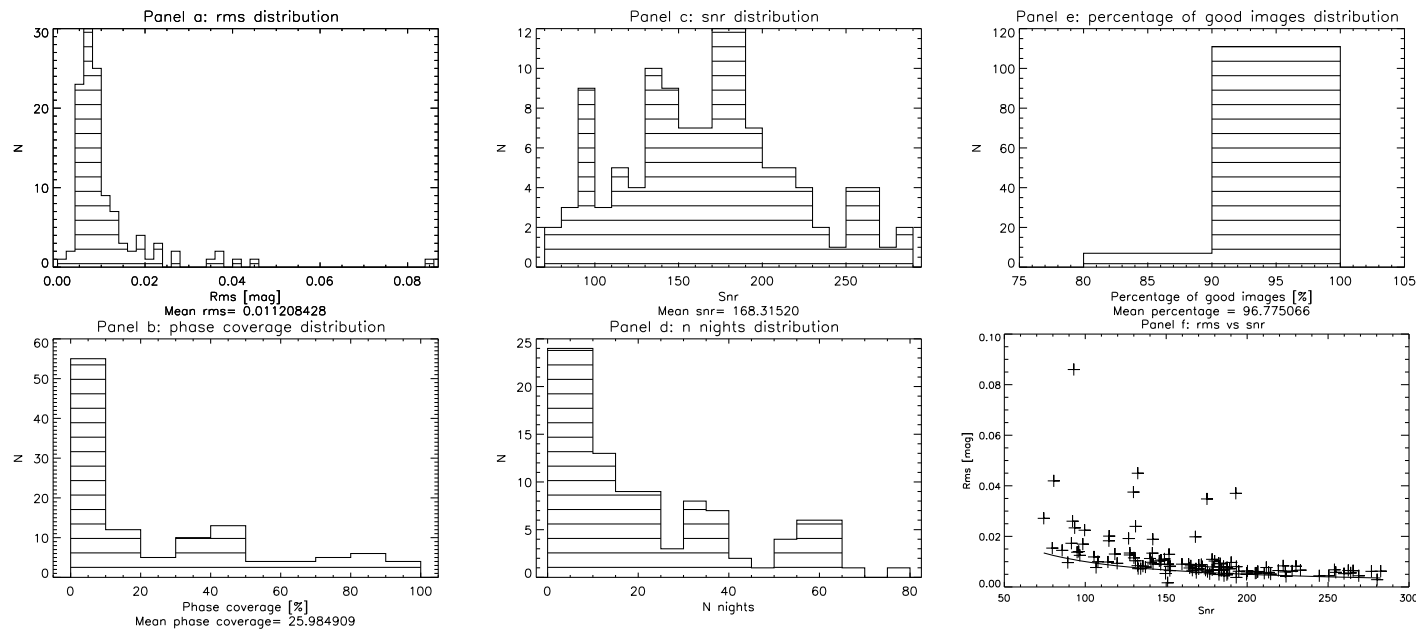

Figure 2. Panel a) mean rms distribution over the observed sample of targets. Panel b) phase coverage distribution over the observed sample of targets for orbital period in the range 0.5-5 days. Panel c) attended Snr distribution over the observed sample of targets. Panel d) number of nights distribution over the observed sample of targets. Panel e) percentage of good images over the whole raw image dataset distribution. Panel f) measured Rms VS Attended Snr over the observed sample of targets (cross), attended Rms VS Attended Snr over the observed sample of targets (solid line).

\section{TOOLS AND HW ORGANIZATION OF APACHE IMAGE ACQUISITION SYSTEM}

We present here the set-up of the hardware (Fig. 1, panel 1) and the software system (Fig. 1, panel 2) built for the APACHE project. The main aim of this part of the project is the HW's and SW's fully robotization. For this purpose every tool is designed for working in total autonomy and for checking itself the coherence of results. The telescope system is fully managed by RTS2 that receives the schedule generated by our tool and then organizes the image acquisition and all the preliminary activities, as dark and flat field acquisition, focusing frames, in automatic way.

\section{FIRST RESULTS FROM THE APACHE OBSERVATIONS}

After 4 months of observations at regime, 93 red dwarfs have been monitored for several nights and some useful results have been obtained, which reveal the good stability of the hardware systems and the encouraging quality of the data.

\section{References}

[1] Damasso et al., PASP 122,895-1077 (2010)

[2] Lepine et Gaidos, AJ 142, art.id. 138 (2011) 